\title{
Neuromonitoraggio intraoperatorio in chirurgia tiroidea: considerazioni nell'ambito della Società Italiana Unitaria Endocrinochirurgia (SIUEC)
}

\author{
Gianlorenzo Dionigi ${ }^{1}$. Pier Giorgio Calò ${ }^{2}$. Gabriele Materazzi ${ }^{3}$. Maurizio lacobone ${ }^{4}$. \\ Nadia Innaro ${ }^{5}$. Maurizio De Palma ${ }^{6}$. Luciano Pezzullo ${ }^{7}$ Maria Grazia Chiofalo ${ }^{7}$. \\ Alberto Zaccaroni ${ }^{8}$. Antonella Pino ${ }^{1}$. Paolo Carcoforo ${ }^{9}$. Annamaria D'Amore ${ }^{10}$. \\ Carmela De Crea ${ }^{10}$. Nicola Avenia ${ }^{11}$. Marco Raffaelli ${ }^{10}$. Giovanni Conzo ${ }^{12}$. Paolo Del Rio ${ }^{13}$. \\ Giovanni Docimo ${ }^{14}$. Mario Testini ${ }^{15}$. Celestino Pio Lombardi ${ }^{10}$ \\ Accettato: 2 aprile 2020 \\ (c) Springer Nature Switzerland AG
}

Sommario L'identificazione routinaria e la visualizzazione diretta del nervo laringeo ricorrente (NLR) sono riconosciute in tutto il mondo come i gold standard nella tecnica di dissezione della tiroide. Il neuromonitoraggio intraoperatorio (IONM) può completare l'informazione offerta dall' $i$ dentificazione diretta attraverso un'analisi funzionale. L'e-

Proposto da Gianlorenzo Dionigi.

Materiale elettronico supplementare La versione elettronica di questo articolo (https://doi.org/10.1007/s40619-020-00779-z) contiene materiale supplementare, disponibile per gli utenti autorizzati.

\section{G. Dionigi}

gdionigi@unime.it

1 Divisione di Chirurgia Endocrina e Mininvasiva, Università degli Studi di Messina, Messina, Italia

2 Dipartimento di Chirurgia, Università di Cagliari, Monserrato, Cagliari, Italia

3 Divisione di Chirurgia, Università di Pisa, Pisa, Italia

4 Divisione di Endocrinochirurgia, Dipartimento di Chirurgia, Università di Padova, Padova, Italia

5 Divisione di Chirurgia, Università Magna Grecia, Catanzaro, Italia

6 Divisione di Chirurgia, Azienda Ospedaliera di Rilievo Nazionale A. Cardarelli, Napoli, Italia

7 Divisione Chirurgia Tiroide e Paratiroide, IRCCS G. Pascale, Napoli, Italia

8 Divisione Chirurgia Endocrina, AUSL Romagna-Morgagni-Pierantoni, Forlì, Italia

9 Dipartimento di Chirurgia, Università di Ferrara, Ferrara, Italia

10 Università Cattolica del Sacro Cuore, Roma, Italia

11 Divisione Chirurgia Endocrina, Università di Perugia, Perugia, Italia

12 Dipartimento di Scienze Mediche, Chirurgiche, Neurologiche, sperienza oramai decennale in Italia con il neuromonitoraggio ha notevolmente contribuito a una più estesa comprensione della fisiopatologia della paralisi del NLR. Il vantaggio principale dello IONM, tuttavia, è la possibilità di riconoscere una paralisi del NLR visivamente non rilevabile, identificando i potenziali meccanismi di danno e, talvolta, riducendo il rischio di un danno nervoso bilaterale. Poiché la perdita del segnale intraoperatorio può richiedere una modifica della strategia chirurgica preoperatoriamente pianificata, le presenti raccomandazioni forniscono informazioni sulle possibilità e sui limiti dello IONM nel momento decisionale di una perdita del segnale intraoperatoria. Ci si augura, comunque, che ulteriori studi, il progressivo affinamento della tecnologia e la corretta applicazione dello IONM nella chirurgia tiroidea si evolvano in modo significativo nei prossimi anni.

Parole chiave Tiroide Chirurgia $\cdot$ Nervo ricorrente . Monitoraggio $\cdot$ Complicanze

\section{Introduzione}

Il neuromonitoraggio intraoperatorio (IONM) nella chirurgia della tiroide è diventato sempre più popolare in Italia $\mathrm{e}$

Metaboliche e dell'Invecchiamento, Università della Campania Luigi Vanvitelli, Caserta, Italia

13 Dipartimento di Chirurgia, Università di Parma, Parma, Italia

14 Dipartimento di Scienze Cardiotoraciche e Respiratorie, Università della Campania Luigi Vanvitelli, Caserta, Italia

15 Divisione Chirurgia Universitaria “V. Bonomo", Dipartimento di Scienze Biomediche ed Oncologia Umana, Università di Bari, Bari, Italia 


\section{della procedura [3].}

Poiché la dissezione sincrona alla stimolazione dei NLR non è ad oggi possibile con la tecnologia del dispositivo IONM disponibile, le aspettative inizialmente stabilite su questa tecnica non sono state in grado di condurre a una significativa riduzione del tasso di paralisi del NLR rispetto alla sola visualizzazione diretta [4]. Pertanto, lo IONM non sostituisce l'identificazione e esposizione del NLR, che sono ancora i requisiti di base.

Se le nuove tecniche di neuromonitoraggio intraoperatorio continuo (C-IONM) [5] possano prevenire danni in futuro e quindi ridurre il rischio di paresi, dovrà essere chiarito da studi appropriati. Le presenti raccomandazioni si riferiscono, quindi, esclusivamente allo stato attuale della tecnica di applicazione del neuromonitoraggio intermittente.

\section{R1. Nel neuromonitoraggio intermittente, la dissezione e la} stimolazione del NLR non possono avvenire contemporaneamente ma in sequenza. Pertanto, anche con l'utilizzo del IONM, la paralisi del NLR non può essere evitata.

\section{Obiettivi}

Sulla base di numerosi studi, ci sono prove sufficienti che lo IONM può essere utilizzato per confermare l'identificazione del NLR e per prevedere la funzione postoperatoria della motilità delle corde vocali [6]. Il raggiungimento di entrambi gli obiettivi dello IONM si basa sull' accuratezza funzionale della tecnologia del dispositivo IONM disponibile. Tuttavia, questi obiettivi possono essere raggiunti in modo affidabile solo se la piena funzionalità della corda vocale è stata dimostrata prima dell'intervento e lo IONM è usato in conformità agli standard [7]. La valutazione elettrofisiologica della funzione del NLR richiede un'elettromiografia (EMG) dei segnali di stimolazione per distinguere gli artefatti [8].

Lo IONM non fornisce informazioni attendibili nella paralisi del NLR preoperatoria, poiché circa il $10 \%$ delle corde vocali paretiche preesistenti ha un segnale EMG sul NV quasi normale. Inoltre, lo IONM dei NV e NLR non è sufficientemente affidabile per la diagnosi funzionale intraoperatoria della branca esterna del nervo laringeo superiore (BENLS), poiché in quest'ultimo caso l'organo da cui deriva il segnale elettromiografico non è un muscolo vocale delle corde vocali, ma il muscolo cricotiroideo, e una branca nervosa comunicante esiste solo in circa il 70-80\% dei casi [9].

Sulla base di studi esistenti si può affermare che vi sia una motilità intatta della corda vocale ipsilaterale nel postoperatorio nel $>97 \%$ (vero negativo) in caso di un segnale EMG intraoperatorio presente sui NV e NLR prima e dopo la resezione [10]. La precisione predittiva nella perdita del segnale intraoperatorio, d'altra parte, è inferiore (60-90\%) a causa di possibili fattori di confondimento che possono portare alla perdita del segnale [11].

R2. Lo IONM del nervo laringeo inferiore è indicativo quando la motilità della corda vocale preoperatoriamente è intatta.

R3. Una valutazione funzionale della BENLS è possibile se è presente il ramo comunicante (70-80\%). Oppure, la stimolazione diretta del BENLS determina una curva elettromiografica e simultaneamente una contrazione (twitch) del muscolo cricotiroideo ipsilaterale.

R4. L'EMG viene utilizzato per confrontare la funzione del NLR pre-e post-dissezione. La documentazione EMG deve essere conservata.

\section{Standard applicativi}

\section{Tecnologia dei dispositivi}

Con funzione nervosa intatta, l'EMG dei muscoli della corda vocale (mediata dal NV e dai NLR) viene trasformata in un segnale acustico. L'EMG dei muscoli della corda vocale è registrato da elettrodi e visualizzato sul monitor [12]. I dispositivi IONM senza rappresentazione EMG, esclusivamente acustici, non consentono una chiara distinzione tra un segnale di artefatto e un potenziale d'azione dei muscoli delle corde vocali. Il tono udibile può essere valutato solo in combinazione con un segnale EMG di stimolazione del NV intatto come prova della presenza di una funzione del NLR. Pertanto, oggi sono preferibili sistemi IONM con rappresentazione grafica del EMG.

L'elettrodo per la registrazione EMG dei muscoli della corda vocale è posizionato o direttamente tramite elettrodi ad ago, che sono introdotti nel legamento cricotiroideo, o indirettamente tramite elettrodi di superficie sul tubo endotracheale, che registrano il potenziale di azione muscolare della contrazione delle corde vocali.

I vantaggi dell'elettrodo ad ago sono la risposta del segnale significativamente più forte e indipendente dalla posizione del tubo. Gli svantaggi sono l'invasività e la derivazione unilaterale che, nel caso della resezione bilaterale, richiede il riposizionamento dell'ago anche controlaterale.

Al contrario, i vantaggi dell'elettrodo di superficie sul tubo endotracheale sono la sua non invasività e l'opzione di derivazione bilaterale. Gli svantaggi sono la risposta di 
stimolazione più bassa e la dipendenza dalla posizione del tubo [13].

R5. Uno IONM adeguato è possibile sia con elettrodi di superficie applicati al tubo endotracheale che con elettrodi ad ago.

R6. L'EMG dei muscoli delle corde vocali è una parte indispensabile del monitoraggio intermittente. I segnali artefatto possono essere differenziati dai veri potenziali d'azione solo dal corrispondente EMG.

\section{Laringoscopia preoperatoria (L1)}

La laringoscopia preoperatoria (L1) è un prerequisito per lo IONM sia negli interventi primari che nelle recidive.

R7. La laringoscopia preoperatoria è richiesta per l'interpretazione dello IONM, in quanto la paralisi del NLR preesistente può essere associata a normale EMG.

\section{Anestesia}

Per un'adeguata neurostimolazione intraoperatoria è necessaria un'induzione anestesiologica che non preveda il miorilassamento. L'induzione e l'intubazione della narcosi sono quindi adattate al peso con rilassanti a breve durata d'azione [14]. Sono disponibili diverse varianti di tubi endotracheali con elettrodi di derivazione EMG [15]:

1. tubi su cui vengono applicati manualmente i collegamenti autoadesivi;

2. tubi prefabbricati con elettrodi di superficie.

Decisiva nella scelta del tubo è una dimensione del tubo adeguata, poiché solo con un buon contatto del tubo con la mucosa laringea a livello della corda vocale è possibile uno IONM affidabile. L'impedenza visualizzata sul monitor indica il contatto tra l'elettrodo del tubo e la mucosa delle corde vocali, dovrebbe essere $<5 \mathrm{k} \Omega$ e la differenza dovrebbe essere $<1 \mathrm{k} \Omega$. Importante per il posizionamento e il fissaggio adeguati del tubo è la collocazione, sotto diretta visione, degli elettrodi al livello delle corde vocali. Poiché la posizione del tubo può spostarsi dopo l'iperestensione del collo, potrebbe essere necessario riposizionare il tubo [16].

R8. Per un'adeguata neurostimolazione intraoperatoria, ̀̀ necessaria narcosi priva di miorilassamento durante la fase dello IONM.

R9. Se si utilizzano tubi endotracheali elettrodi di superficie, le dimensioni e il posizionamento appropriati dei tubi endotracheali richiedono un adeguato contatto della mucosa (impedenza) della corda vocale.

\section{Stimolazione vagale pre-dissezione (V1)}

Quando la motilità della corda vocale è conservata, la stimolazione del NV diretta preliminare (V1) verifica la funzione del NLR lungo tutto il suo decorso anatomico e deve quindi essere eseguita prima della preparazione del NLR [17].

Per la stimolazione durante tutta la procedura chirurgica, si consiglia una corrente sovramassimale di 1-2 mA [18].

Per la stimolazione vagale iniziale, il NV, fisiologicamente collocato tra la giugulare interna e l'arteria carotide comune deve essere identificato il più caudalmente possibile, specialmente sul lato destro, per la rara variante anatomica (circa $0,5 \%$ ) di un nervo laringeo inferiore non ricorrente sul lato destro [19]. La presenza di un nervo laringeo inferiore non ricorrente è tipicamente evidente in assenza del segnale di stimolazione vagale più caudale. Se si sospetta un nervo laringeo inferiore non ricorrente, il nervo vago deve essere stimolato cranialmente il più possibile, dopo la stimolazione caudale primaria.

I parametri del segnale EMG sul NV pre-dissezione (ampiezza, latenza, forma d'onda) sono riferimenti per l'identificazione del NLR, la dissezione del NLR e i cambiamenti del segnale EMG durante l'intervento chirurgico. All'inizio dell'intervento chirurgico, la massima ampiezza possibile dovrebbe essere raggiunta sotto la stimolazione sopramassimale. Lorenz e collaboratori hanno riportato valori predefiniti del nervo vago sinistro e nervo vago destro $>300 \mu \mathrm{V}$ come riferimento [20].

Se l'ampiezza è insufficiente, è necessaria una correzione della posizione del tubo endotracheale quando si utilizzano gli elettrodi adesi al tubo endotracheale; la correzione del posizionamento dell'ago è necessaria quando si utilizzano gli elettrodi dell'ago.

Alla stimolazione del NV, l'evidenza palpatoria del laryngeal twich può essere indicativa di funzione nervosa ipsilaterale del NLR conservata $[4,20]$. Tuttavia, non sono disponibili studi sistematici sulla correlazione della contrazione laringea con EMG e funzionalità postoperatoria.

R10. È necessaria una stimolazione vagale preliminare per monitorare la funzione nervosa del NLR durante l'intero corso della procedura.

R11. In caso di stimolazione vagale caudale e assenza del segnale di stimolazione vagale, il neuromonitoraggio può presto indicare la rara variante di un nervo laringeo inferiore non ricorrente sul lato destro.

R12. L'ampiezza del segnale iniziale sul nervo vago deve essere di almeno $300 \mu \mathrm{V}$ con stimolazione sopramassimale di 1,0-2,0 mA, altrimenti si consiglia la correzione della posizione del tubo o dell'ago.

\section{Stimolazione del nervo laringeo ricorrente pre-dissezione (R1)}

La stimolazione preliminare diretta del nervo ricorrente (R1) conferma la struttura visivamente identificata del NLR valutandone anche la funzionalità. Tramite la stimolazione nervosa diretta, le strutture non nervose possono essere discriminate, specialmente nel caso di re-interventi in cui vi siano 
esiti cicatriziali che scompaginano l'anatomia, e può essere effettuata una determinazione iniziale della posizione dei nervi ("mappatura") [4]. La stimolazione del NV e del NLR pre-dissezione funge da riferimento funzionale per eventuali cambiamenti nella conduzione nervosa durante la resezione.

I parametri della stimolazione intermittente diretta del NLR di solito mostrano un'ampiezza maggiore rispetto alla stimolazione del NV ipsilaterale [19].

\section{R13. La stimolazione del nervo laringeo inferiore funge da riferimento funzionale per eventuali cambiamenti nella conduzione nervosa durante la resezione.}

\section{Mapping decorso nervoso}

Il cosiddetto mapping del NLR descrive il processo ripetuto di stimolazione intermittente lungo il suo probabile decorso [4]. Lo scopo del mapping è di anticipare, talvolta, il decorso del nervo non ancora anatomicamente esposto al fine di eseguire il successivo processo di identificazione visiva e conferma elettrofisiologica del NLR e, quindi, resezione tiroidea, sotto diretta visione, e conservazione del NLR. Nel caso di manovre chirurgiche nelle immediate vicinanze del nervo il feedback nervoso esclusivamente funzionale non è sufficiente, e la dissezione vicino al nervo richiede pertanto una chiara e diretta visione.

Per la stimolazione del NLR sono disponibili vari modelli di sonda. Le sonde di stimolazione unipolare hanno un raggio d'azione più ampio rispetto alle sonde di stimolazione bipolare. Queste ultime sono quindi più efficaci nei casi di difficoltosa identificazione del nervo anatomico, per la loro più precisa discriminazione tra il nervo e le strutture non neuronali.

Come nel caso della stimolazione intermittente iniziale del NLR, anche nel corso dell'intervento chirurgico è richiesta una differenziazione affidabile tra segnali di artefatto e i reali potenziali di azione nervosa, al fine di essere in grado di distinguere in modo affidabile strutture nervose, non nervose e una funzionalità alterata. I segnali di artefatto nell'EMG possono essere riconosciuti dalla mancanza di latenza tra stimolazione e insorgenza del potenziale di azione del nervo [3]. Al solo segnale acustico i segnali di artefatto sono indistinguibili dai veri potenziali di azione.

\section{R14. Il mapping del NLR descrive il processo ripetuto di} stimolazione intermittente lungo il suo probabile decorso. Lo scopo del mapping è di anticipare il decorso del nervo non ancora anatomicamente esposto al fine di eseguire il successivo processo di identificazione visiva e conferma elettrofisiologica del NLR e quindi resezione tiroidea, sotto diretta visione, e conservazione del NLR.

R15. Entrambe le sonde di stimolazione, unipolare e bipolare, sono adatte, tenendo conto della loro diversa capacità di discriminazione.
Simulazione del vago post-dissezione (V2)

Una stimolazione del vago finale (V2) viene eseguita al completamento di tutte le procedure chirurgiche sul lato operato e il risultato viene documentato e archiviato. La stimolazione vagale $\mathrm{V} 2$ rende possibile, con elevata probabilità, prevedere il mantenimento funzionale del NLR. Nel caso di un intervento chirurgico bilaterale, il risultato V2 è un prerequisito per l'indicazione per l'operazione del lato controlaterale [8].

R16. Quando il NLR, dopo la resezione, è stato preservato, il risultato della stimolazione vagale (V2) ne mostra l'attuale funzionalità e influenza la decisione su come procedere sul lato controlaterale.

\section{Laringoscopia postoperatoria (L2)}

La valutazione laringoscopica postoperatoria riguardo la motilità delle corde vocali è il gold standard per la valutazione della loro funzione ed è quindi essenziale per la diagnosi funzionale e il controllo di qualità [5]. In rari casi, la funzione della corda vocale valutata laringoscopicamente su pazienti coscienti e cooperativi si discosta dal risultato dell'IONM.

R17. La valutazione laringoscopica della motilità delle corde vocali è il gold standard postoperatorio nonché preoperatorio di diagnostica della funzionalità delle corde vocali e, quindi, una componente obbligatoria della diagnostica e del controllo di qualità dopo un intervento alla tiroide.

\section{Procedura in caso di perdita del segnale intraoperatorio}

La perdita di segnale intraoperatoria è definita come una perdita completa (perdita di segnale). Quando si utilizza un elettrodo di superficie, con l'attuale tecnologia del dispositivo IONM, una caduta di ampiezza a $<100 \mu \mathrm{V}$ nella stimolazione del NV si traduce, con alta probabilità $(>90 \%)$, in perdita di funzionalità del nervo stimolato [4].

Supponendo che la motilità della corda vocale preoperatoriamente sia conservata, la seguente correlazione tra la risposta del segnale intraoperatorio e la motilità ipsilaterale postoperatoria della corda vocale risulta dai seguenti risultati $[9,20]$ :

1. vero negativo: segnale EMG vagale intatto, mobilità postoperatoria ipsilaterale intatta;

2. falso negativo: segnale EMG vagale intatto, paralisi cordale postoperatoria ipsilaterale;

3. vero positivo: perdita di segnale intraoperatoria, paralisi postoperatoria ipsilaterale della corda vocale; 
L'Endocrinologo

Fig. 1 Algoritmo per la gestione della perdita di segnale intraoperatorio e valutazione del tipo di lesione.

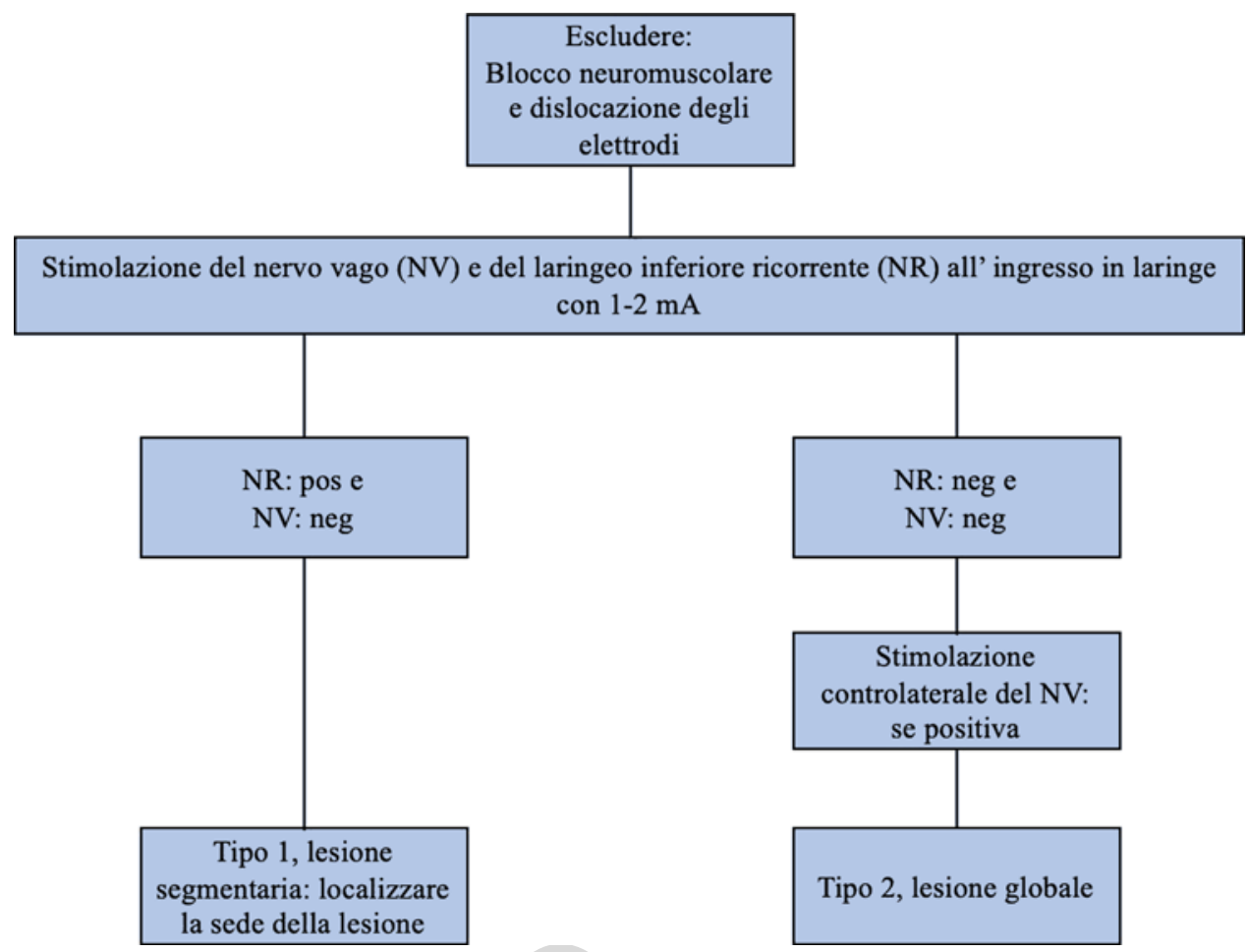

Tabella 1 Correlazione della risposta del segnale intraoperatorio e del risultato postoperatorio (motilità cordale) e con le possibili cause di risultati falsi positivi e falsi negativi

\begin{tabular}{ll}
\hline Risultati & Possibili cause \\
\hline - Vero negativo (segnale EMG & \\
sul nervo vago intatto, mobilità & \\
della corda vocale intatta) & \\
- Falso negativo (segnale EMG & - Cause extraneurali \\
sul nervo vago intatto, paralisi & - Disturbi nervosi centrali \\
della corda vocale) & \\
- Vero positivo (perdita del & \\
segnale, paralisi della corda & \\
vocale) & \\
- Falso positivo (perdita di & - Paralisi farmacologica del nervo \\
segnale, mobilità della corda & vago (anestesia-correlato) \\
vocale intatta) & - Stimolazione nervosa inadeguata \\
& - Disfunzione dispositivi \\
& - Recupero precoce della funzione \\
& nervosa \\
\hline
\end{tabular}

4. falso positivo: perdita di segnale intraoperatoria, motilità ipsilaterale postoperatoria conservata.

Le possibili cause di risultati falsi negativi e falsi positivi sono indicate nella Tabella 1. Una paralisi del NLR che perduri più di 6 mesi è altamente suggestiva di paralisi permanente.

R18. Una caduta di ampiezza inferiore a $100 \mu \mathrm{V}$ del segnale vagale precedentemente intatto e quantitativamente normale rende molto probabile una perdita di funzionalità del nervo stimolato. In caso di perdita del segnale o caduta di ampiezza inferiore a $100 \mu \mathrm{V}$, è possibile una previsione errata della mobilità postoperatoria della corda vocale in circa il $10 \%$ per diversi

R19. In correlazione con il parametro di riferimento della motilità della corda vocale preoperatoria e postoperatoria, il segnale vagale intatto invariato e l'EMG al termine della resezione (V2) in oltre il $97 \%$ consentono una previsione corretta della funzione della corda vocale postoperatoria.

Per ridurre i risultati falsi negativi o falsi positivi, la Figura 1 mostra un algoritmo utile per i chirurghi. In seguito a una perdita di segnale del $\mathrm{NV}$, dopo il controllo del dispositivo IONM e l'esclusione di un blocco neuromuscolare indotto dagli anestetici, si dovrebbe prima verificare se vi è una dislocazione del tubo endotracheale o dell'elettrodo ad ago. Se c'è un segnale positivo sul NLR dopo un stimolazione NV negativa, c'è un danno nervoso localizzato prossimalmente al punto di stimolazione (lesione tipo 1, "segmentaria", Fig. 2) [3]. Se non vi è alcun segnale di stimolazione nervosa in caso di stimolazione NLR e NV, si può ipotizzare un disturbo della conduzione nervosa globale (tipo 2, Fig. 2).

Nel caso di una lesione ricorrenziale di tipo 1 ("segmentaria"), la mappatura retrograda del nervo laringeo ricorrente può, se necessario, localizzare il sito della lesione con l'obiettivo di identificare la potenziale causa del disturbo motivi. 
Tipo 1, segmentaria

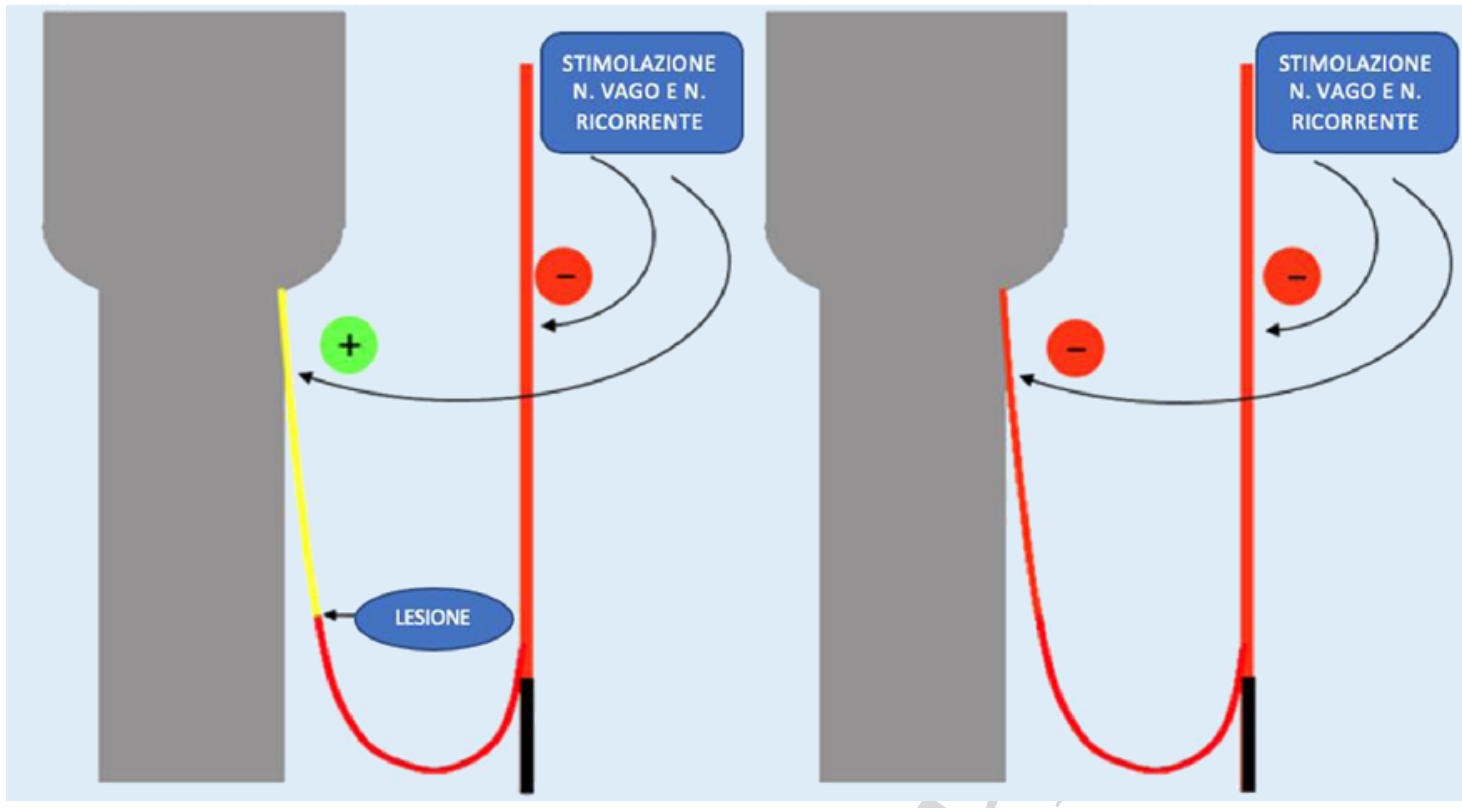

Fig. 2 Valutazione e definizione della perdita di segnale intraoperatorio secondo il tipo 1 (lesione segmentaria) o il tipo 2 (lesione globale).

neurologico e, qualora possibile, eliminarla. I corticosteroidi possono essere somministrati per via endovenosa o topica in entrambe le forme di disfunzione nervosa [7]. Tuttavia, non vi sono dati sufficientemente affidabili sulla dose ottimale e sui risultati della somministrazione di steroidi, quindi non sono possibili raccomandazioni terapeutiche appropriate.

R20. Per ridurre i risultati IONM falsi positivi e falsi negativi, proponiamo un algoritmo da adottare, utile in caso di perdita del segnale intraoperatorio, che può quindi distinguere tra un danno localizzato (tipo 1) $o$ globale (tipo 2) del NLR.

R21. Se la somministrazione di corticosteroidi promuova la rigenerazione del danno ai nervi quando si è verificata la perdita del segnale non è ancora adeguatamente confermato dagli studi.

\section{Cambio di strategia chirurgica in caso di perdita del segnale}

Nel caso di gozzo multinodulare benigno e di chirurgia programmata bilaterale, di volta in volta può essere preso in considerazione un cambio di strategia quando si verifica la perdita di segnale sul primo lato approcciato [5, 20]. L'obiettivo del cambio di strategia è la protezione sicura della funzionalità del NLR sul lato controlaterale. L'opzione più sicura è quella di rinunciare completamente alla resezione del lato controlaterale in questa situazione. I rischi di una procedura in due fasi dopo il recupero del- la funzione di piega vocale sono generalmente considerati inferiori al potenziale rischio di una paralisi ricorrenziale bilaterale.

Se, in presenza di un tumore alla tiroide, deve essere preso in considerazione un cambiamento di strategia con una procedura sequenziale in due tempi, tenendo conto dei vantaggi e dei rischi, dipende dal tipo del tumore e richiede una valutazione caso per caso, preoperatoria, ampiamente discussa con il paziente, come da consenso informato, l'anestesista e l'endocrinologo.

R22. Per il gozzo benigno e la tiroidectomia totale pianificata si consiglia un cambio di strategia se viene registrata una perdita di segnale sul primo lato resecato dominante.

R23. In caso di neoplasia maligna e di una perdita di segnale sul lato operato per primo, la decisione su una resezione controlaterale a uno o due stadi dipende dal tipo del tumore e richiede una valutazione caso per caso, preoperatoria, ampiamente discussa con il paziente, l'endocrinologo e l'anestesista.

\section{Informazione, documentazione}

\section{Informazione preoperatoria}

Lo scopo dell'informazione preoperatoria è di spiegare al paziente il significato e i limiti della procedura, specialmente nel caso di un'indicazione chirurgica per una procedura bilaterale. 
Lo IONM può riconoscere i disturbi funzionali rilevanti del nervo laringeo ricorrente anche senza una correlazione anatomica visibile. La perdita del segnale intraoperatorio e l'attenuazione significativa del segnale sono molto probabilmente associate alla paralisi (precoce) postoperatoria della corda vocale. Dopo la perdita bilaterale del segnale intraoperatorio, quindi, il rischio di paresi bilaterale è più elevato. Per evitare al meglio la paralisi bilaterale si raccomanda, pertanto, di valutare, caso per caso, l'opportunità di un cambiamento di strategia per quanto riguarda la resezione dell'altro lato dopo la perdita del segnale, omettendo eventualmente la resezione simultanea del lato opposto.

Tuttavia, un risultato falso positivo dello IONM (perdita del segnale intraoperatorio, motilità conservata) può significare che la resezione del lato opposto avviene solo in una seconda procedura. La probabilità di risultati falsi positivi del neuromonitoraggio è attualmente del 10-30\% [3].

$\mathrm{Al}$ contrario, un risultato falso negativo (segnale intraoperatorio normale, paralisi cordale postoperatoria) può portare a paralisi ricorrenziale bilaterale nonostante l'uso della procedura. La probabilità di risultati falsi negativi del neuromonitoraggio con la conseguenza della paresi bilaterale ricorrente è molto bassa $(<1 \%)$, ma è stata descritta in letteratura $[2,9]$.

\section{Documentazione}

Per quanto riguarda la documentazione, la chirurgia della tiroide che prevede l'utilizzo dello IONM va oltre la documentazione della diretta visione del nervo, in cui è sufficiente la descrizione scritta della visualizzazione e del risparmio del nervo prima, durante e dopo la resezione tiroidea. Come per l'uso di altri ausili operativi, è consigliabile anche la documentazione per la tecnica IONM [7]. Solo la stimolazione EMG può identificare in sicurezza un artefatto da un vero potenziale di azione $[2,8]$.

Il requisito minimo della documentazione del neuromonitoraggio è la descrizione dei segnali di stimolazione del nervo vago sul lato operato dopo il completamento di tutte le misure chirurgiche nella loggia tiroidea (V2).

R24. Il requisito minimo per la documentazione del neuromonitoraggio è la descrizione dei segnali di stimolazione del nervo vago sul lato operato dopo il completamento di tutte le manovre chirurgiche (V2).

Conflitto di interesse Gli autori Gianlorenzo Dionigi, Pier Giorgio Calò, Gabriele Materazzi, Maurizio Iacobone, Nadia Innaro, Maurizio De Palma, Luciano Pezzullo, Maria Grazia Chiofalo, Alberto Zaccaroni, Antonella Pino, Paolo Carcoforo, Annamaria D'Amore, Carmela De Crea, Nicola Avenia, Marco Raffaelli, Giovanni Conzo, Paolo Del Rio, Giovanni Docimo, Mario Testini e Celestino Pio Lombardi dichiarano di non avere conflitti di interesse.

Consenso informato Lo studio presentato in questo articolo non ha richiesto sperimentazione umana.
Studi sugli animali Gli autori di questo articolo non hanno eseguito studi sugli animali.

Nota della casa editrice Springer Nature rimane neutrale in riguardo alle rivendicazioni giurisdizionali nelle mappe pubblicate e nelle affiliazioni istituzionali.

\section{Bibliografia}

1. Randolph GW, Dralle H, Abdullah H et al (2011) Electrophysiologic recurrent laryngeal nerve monitoring during thyroid and parathyroid surgery: international standards guideline statement. Laryngoscope 121:S1-S16

2. Dralle H, Sekulla C, Lorenz K et al (2012) Loss of the nerve monitoring signal during bilateral thyroid surgery. Br J Surg 99:1089-1095

3. Brauckhoff M, Gimm O, Nguyen Thanh P et al (2002) First experiences in intraoperative neurostimulation of the recurrent laryngeal nerve during thyroid surgery of children and adolescents. J Pediatr Surg 37:1414-1418

4. Dralle H, Sekulla C, Haerting J et al (2004) Risk factors of paralysis and functional outcome after recurrent laryngeal nerve monitoring in thyroid surgery. Surgery 136:1310-1322

5. Dralle H, Sekulla C, Lorenz K et al (2008) Intraoperative monitoring of the recurrent laryngeal nerve in thyroid surgery. World $\mathrm{J}$ Surg 32:1358-1366

6. Barczynski M, Konturek A, Cichon S (2009) Randomized clinical trial of visualization versus neuromonitoring of recurrent laryngeal nerves during thyroidectomy. Br J Surg 96:240-246

7. Alesina PF, Rolfs T, Hommeltenberg S et al (2012) Intraoperative neuromonitoring does not reduce the incidence of recurrent laryngeal nerve palsy in thyroid reoperations: results of a retrospective comparative analysis. World J Surg 36:1348-1353

8. Schneider R, Przybyl J, Hermann M et al (2009) A new anchor electrode design for continuous neuromonitoring of the recurrent laryngeal nerve by vagal nerve stimulations. Langenbeck's Arch Surg 394:903-910

9. Schneider R, Randolph GW, Sekulla C et al (2013) Continuous intraoperative vagus nerve stimulation for identification of imminent recurrent laryngeal nerve injury. Head Neck 35(11):1591-1598

10. Chan WF, Lo CY (2006) Pitfalls of intraoperative neuromonitoring for predicting postoperative recurrent laryngeal nerve function during thyroidectomy. World J Surg 30:806-812

11. Dralle H, Lorenz K (2010) Intraoperatives Neuromonitoring bei Schilddrüsenoperationen. Chirurg 81:612-619

12. Chiang FY, Lee KW, Chen HC et al (2010) Standardization of intraoperative neuromonitoring of recurrent laryngeal nerve in thyroid operation. World J Surg 34:223-229

13. Lorenz K, Sekulla C, Schelle J et al (2010) What are normal quantitative parameters of intraoperative neuromonitoring (IONM) in thyroid surgery? Langenbeck's Arch Surg 395:901-909

14. Barczynski M, Randolph GW, Cernea CR et al (2013) External branch of the superior laryngeal nerve monitoring during thyroid surgery: international standards guideline statement. Laryngoscope 123:1-14

15. Dionigi G, Chiang FY, Rausei S et al (2010) Surgical anatomy and neurophysiology of the vagus nerve $(\mathrm{VN})$ for standardized intraoperative neuromonitoring (IONM) of the inferior laryngeal nerve (ILN) during thyroidectomy. Langenbeck's Arch Surg 395:893-899

16. Brauckhoff M, Walls G, Brauckhoff K et al (2002) Identification of the non-recurrent inferior laryngeal nerve using intraoperative neurostimulation. Langenbeck's Arch Surg 386:482-487 
17. Brauckhoff M, Machens A, Sekulla C et al (2011) Latencies shorter than $3.5 \mathrm{~ms}$ after vagus nerve stimulation signify a nonrecurrent inferior laryngeal nerve before dissection. Ann Surg 253:1172-1177

18. Dionigi G, Boni L, Rovera F et al (2010) Postoperative laryngoscopy in thyroid surgery: proper timing to detect recurrent laryngeal nerve injury. Langenbeck's Arch Surg 395:327-331
19. Dralle H, Lorenz K, Andreas M (2012) Verdicts on malpractice claims after thyroid surgery: emerging trends and future directions. Head Neck 34:1591-1596

20. Melin M, Schwarz K, Lammers BJ et al (2013) IONM-guided goiter surgery leading to two-stage thyroidectomy-indication and results. Langenbeck's Arch Surg 398:411-418 